\title{
Implantatentfernung beim Kind
}

\author{
Stefan Nuber
}

\section{Zusammenfassung}

Derzeit werden aufgrund v.a. gestiegener Ansprüche, wie z.B. der Wunsch einer gipsfreien Behandlung, häufiger primäre Osteosynthesen bei kindlichen Frakturen vorgenommen. Dies führt zu einer hohen Anzahl von anschließenden Implantatentfernungen (ME) bei Kindern, ohne dass für diese Eingriffe evidenzbasierte Daten für deren Notwendigkeit vorliegen. So gelten das wachsende Skelett, eine mögliche Implantatkorrosion und v.a. der Wunsch der Eltern als Gründe für die ME bei Kindern. Dem gegenüber stehen die operations- und narkosebedingten Risiken dieses Eingriffs. Letztlich erfolgt die Implantatentfernung bei Kindern nach einer individuellen Indikationsstellung unter Einbeziehung der Eltern. Wie bei den Erwachsenen ist auch bei der ME bei Kindern ein standardisier- tes präoperatives wie auch postoperatives Management notwendig. Neben einem entsprechenden ME-Instrumentarium sind auch eine kindgerechte peri- und postoperative Betreuung und Überwachung erforderlich. Die notwendigen Schritte im Rahmen der Planung, Durchführung und Nachsorge sind zur Realisierung einer erfolgreichen Implantatentfernung bei Kindern ebenso zu beachten, wie auch der Umstand, dass bereits durch die ursprüngliche Frakturbehandlung eine technisch schwere ME möglichst vermieden oder u.U. sogar unnötig wird.

\section{Implant Removal in Children}

The incidence of primary osteosynthesis in child fractures is increasing, due to increasing demand, including the preference for cast-free treatment. This then causes a rise in implant removal procedures in child patients, without evidence that these procedures are required. Skeletal growth, the possibility of implant corrosion and especially parental wishes are considered reasons for implant removal. On the other hand, there are surgical as well as anaesthesiological risks. The indication for implant removal in children must be an individual decision, incorporating the parents. As in adult patients, children require standardised perioperative management for implant removal. Aside from the surgical instruments for the removal, it is also necessary to provide appropriate peri- and postoperative care and monitoring. For successful implant removal in children, careful planning, surgery, and aftercare are essential. The original fracture treatment should aim at avoiding a technically difficult implant removal, if at all possible.

\section{Einleitung}

Jährlich werden in Deutschland insgesamt etwa 180000 Implantatentfernungen (ME) durchgeführt. Somit ist diese Operation mit ca. 30\% einer der häufigsten elektiven Eingriffe in Deutschland $[3,8]$. Für Erwachsene beschreibt die Literatur für die Implantatentfernung lediglich eine relative und selten zwingende Indikation, welche immer individuell gestellt werden sollte. Es besteht hierfür keine evidenzbasierte Empfehlung [1].

Demgegenüber besteht im Allgemeinen Konsens darüber, dass am wachsenden Skelett die Implantatentfernung erfolgen sollte $[1,2,7]$. So kann es z.B. durch eingebrachtes Osteosynthesematerial an

OP-JOURNAL 2016; 32: 96-104

(c) Georg Thieme Verlag KG Stuttgart · New York DOI http://dx.doi.org/10.1055/s-0042-107041 der Diaphyse zur Störung des periost-endostalen Dickenwachstums am Knochen kommen. Durch epiphysenfugenkreuzende oder -perforierende Implantate kann, wie auch durch die Fugenverletzung selbst, ein (partiell) stimulierendes wie auch hemmendes Längenwachstum resultieren. Aber selbst eine Entfernung epiphysenfugenkreuzender Implantate kann zu einer Stimulation der Fuge führen [5].

Als weiterer Grund für die Sinnhaftigkeit der routinemäßigen Implantatentfernung bei Kindern gilt v.a. die durch die Implantatstahllegierungen hervorgerufene Korrosion und die damit verbundene Abgabe der Korrosionsprodukte an das umliegende Gewebe wie auch an andere Organe. Hierdurch können u.a. sekundäre Allergien und deren Folgen induziert werden [4]. Durch die seit Jahren zumeist eingesetzten Implantate aus entsprechenden Titanlegierungen mit deut- lich höherer Biokompatibilität und Korrosionsresistenz besteht die Möglichkeit einer Osteosynthese mit signifikant höherer Gewebeverträglichkeit. Dennoch konnte selbst bei Titanimplantaten im standardisierten Tiermodell, neben lokalen zellulären inflammatorischen Prozessen an Knochen und Gewebe, bereits nach 16 Wochen in der Milz eine erhöhte Konzentration von Titan und elektronenmikroskopisch Titanpartikel nachgewiesen werden [13]. Die hiermit verbundenen Langzeitauswirkungen konnten nicht untersucht werden.

Wie bei den Erwachsenen gibt es auch bei Kindern Umstände, die für eine Implantatentfernung sprechen:

- implantatbedingte funktionelle Einschränkungen

- lokale Reizungen von Sehnen, Muskeln, Faszien und Gelenken, Irritation von Haut und Subkutangewebe 
- biomechanische Gründe, vor allem an der unteren Extremität (z.B. Verminderung der Elastizität innerhalb der implantatgeschützten Knochenstrecke bei steifen Implantaten) [9]

- Bohrdrähte (mögliche Wanderung)

- Implantat an prominenten Körperstellen (z.B. Olekranon, Epicondylus ulnaris)

- implantatassoziierte Infektionen

- Implantate, die aus dem Körper ragen (z.B. Fixateur externe, zur Vermeidung von Pintrack-Infektionen)

- Wunsch des Patienten bzw. des Erziehungsberechtigten

Der Überlegung, warum man die Implantatentfernung bei Kindern nicht regelhaft durchführen sollte bzw. der Ansatz, dass dieser weitere Eingriff nach der Osteosynthese nicht zwingend notwendig sei, wird in der Literatur kaum Beachtung geschenkt. Es gibt so gut wie keine Untersuchungen, die Gruppen mit ME mit Gruppen ohne ME vergleichen und zudem evidenzbasierte Daten liefern. Eine der wenigen Ausnahmen ist eine retrospektive Studie von Morshed et al., bei der von insgesamt 24 Kindern nach elastisch stabiler intramedullärer Nagelung (ESIN) bei Femurschaftfraktur nur bei Beschwerden die ME durchgeführt wurde. Dies war bei 6 Kindern innerhalb der ersten 15 Monate der Fall. Bei einem 5-Jahres-Follow-up hatten noch 17 der 24 Kinder (72\%) die ESIN in situ, und es konnten keine Unterschiede bez. Funktion und Beschwerden in beiden Gruppen festgestellt werden. Langzeitergebnisse fehlen, sodass weitere kontrollierte Studien mit dieser Fragestellung gefordert wurden [6].

Die vermeintlich leichte ME stellt sich in der Realität häufig schwieriger dar als angenommen. In der Literatur wird durchschnittlich eine Komplikationsrate von $10 \%$ bei kindlichen Implantatentfernungen beschrieben [10]. Selbst wenn man die äußerst komplikationsträchtigen MEs nach Stabilisierung einer Epiphysiolysis capitis femoris (ECF) mit durchschnittlich 34\% abzieht, verbleibt für diesen elektiven Eingriff immer noch eine Komplikationsrate von $6 \%$.

Die beste Metallentfernung ist die, die nicht notwendig ist bzw. wäre. Mittlerweile laufen Versuche und Studien mit bioresorbierbaren Osteosynthesematerialien bei Kindern, hauptsächlich in der kraniofazialen Chirurgie, aber auch in der Kindertraumatologie. So wird in einer retrospektiven Fallserie von Taka- da et al. die Versorgung einer Condylusradialis-Fraktur bei insgesamt 8 Kindern mittels bioresorbierbarer Stifte nach offener Reposition mit anschließender 4wöchiger Ruhigstellung in einer Oberarmschiene beschrieben. Alle Frakturen kamen regelrecht zur Ausheilung, bei einem 3-Jahres-Follow-up zeigten sich weder anatomische noch funktionelle Probleme. Als besondere Vorteile gegenüber der standardisierten Versorgung mittels nicht resorbierbaren Osteosynthesematerialien wurden besonders die „freie“ Sicht in der Röntgenkontrolle sowie die nicht notwendige ME angegeben [12]. Eine weitere Arbeitsgruppe aus Finnland um Sinikumpu beschreibt 2013 eine Fallserie von 3 Kindern zwischen 5 und 15 Jahren, die bei einer Unterarmschaftfraktur mittels bioresorbierbarer intramedullärer Nägel ohne Komplikationen versorgt und allesamt zur regelrechten Ausheilung gebracht wurden [11]. Von einem standardisierten Einsatz bioresorbierbarer Osteosynthesematerialien in der Kindertraumtologie kann man jedoch derzeit noch nicht sprechen.

Letztendlich gibt es in der Literatur weder eine Evidenz für noch gegen eine routinemäßige Implantatentfernung bei Kindern [10].

\section{Vorgehen}

Besteht bei einem Kind der Zustand nach Osteosynthese einer Fraktur, so werden im Rahmen der Nachsorge mittels klinischer und radiologischer Kontrolle die Ausheilung der ehemaligen Fraktur und das klinische Outcome festgestellt.

Um die Indikation zur Implantatentfernung stellen zu können, wird eine Anamnese von möglichen und evtl. die Operation einschränkenden Vorerkrankungen und Begleitverletzungen sowie die Dokumentation wichtiger Begleitumstände (Mobilität, soziale Situation, Medikamente) durchgeführt. Bei der klinischen Untersuchung wird der aktuelle postoperative Befund mit u.U. vorliegenden, implantatbedingten oder lokalen Beschwerden, Funktionseinschränkungen oder Fremdkörpergefühl erhoben. Daneben bedarf es der durch Inspektion und Palpation erhobenen Befunde wie vorbestehender OP-Zugang, Narbenbeschaffenheit, Hautverhältnisse, Durchblutung, Motorik, Sensibilität, Zeichen einer Infektion oder Allergie, Funktion des betroffenen Gelenks bzw. der angrenzenden Gelenke, mögliche Achsabweichungen oder Längenunterschiede, mögliche
Instabilitäten, muskuläre Defizite sowie das Gewebegleiten über den einliegenden Implantaten. Eine entsprechende Dokumentation ist obligat.

Vor der Implantatentfernung wird zusätzlich eine zeitnahe Röntgenuntersuchung in 2 Ebenen mit Darstellung sowohl des Frakturbereichs als auch der Implantate in ganzer Ausdehnung zwingend gefordert. Hierdurch muss die Frakturheilung (tragfähiger Kallus), die Art des Implantats, die Zahl der Implantate, ein Implantatbruch, eine -lockerung, -wanderung oder -perforation, ektope Ossifikationen oder eine Überknöcherung des Implantats sicher beurteilt werden können.

Vor allem bei erheblich dislozierten ellenbogennahen Frakturen im Kindesalter (v.a. suprakondyläre Oberarmfrakturen) kommt es immer wieder zu posttraumatischen wie auch iatrogenen Nervenläsionen. Die hiervon betroffenen Kinder sollten prinzipiell im Rahmen der Nachsorge auch an einen Neurologen mit der Möglichkeit der neurophysiologischen Untersuchung sowie auch der Neurosonografie angebunden werden, um das Ausmaß und die Prognose der Nervenläsion abzuschätzen und die Indikation zu einer möglichen operativen Nervenrevision mit dem Neurochirurgen stellen zu können. In diesem Fall ist eine gemeinsame Planung der Implantatentfernung und der Nervenoperation sinnvoll.

Prinzipiell sollte immer der OP-Bericht der Implantation gelesen und bei Bedarf von extern eingeholt werden. Neben der Art sollte auch der Hersteller des Implantats bereits präoperativ bekannt sein, um evtl. notwendige Instrumente rechtzeitig organisieren zu können.

Wird vom behandelnden Arzt die Indikation zur Implantatentfernung bei Kindern gestellt, so muss diese mit den Eltern abgesprochen werden. Prinzipiell ist die Zustimmung beider Elternteile (soweit sorgeberechtigt) erforderlich. Der Arzt kann jedoch bei Routineeingriffen leichterer Art ohne weitreichende Risiken auf die Mitteilung des erschienenen Elternteils vertrauen, zur Zustimmung für den anderen bevollmächtigt zu sein. Andernfalls ist die Einwilligung nur wirksam, wenn beide Elternteile zum Aufklärungsgespräch erscheinen und einwilligen.

Es müssen die vorliegenden Vorteile gegenüber den möglichen Nachteilen dar- 
Tab. 1 Allgemein übliche ME-Zeitpunkte bei radiologisch gesicherter, stabiler knöcherner Konsolidierung.

\begin{tabular}{|c|c|c|c|}
\hline Lokalisation & Implantat & Zeitpunkt & Bemerkung \\
\hline Humerus proximal & ESIN & $3-(6)$ Monate & retrograd \\
\hline \multirow[t]{2}{*}{ Humerusdiaphyse } & ESIN & 3-(6) Monate & retro- u. antegrad \\
\hline & Fixateur externe & 6-8 Wochen & \\
\hline \multirow[t]{2}{*}{ Humerus distal, suprakondylär } & K-Drähte & 3-4 Wochen & transkutan \\
\hline & K-Drähte & 4-6 Wochen & versenkt \\
\hline Humerus distal, epikondylär & Schrauben & 3 Monate & \\
\hline Humerus distal, kondylär & Schraube & 3-4 Monate & \\
\hline Humerus distal, transkodylär & Platte & 6 Monate & \\
\hline Unterarmdiaphyse & ESIN & (3) - 6 Monate & \\
\hline Radius distal & K-Drähte & 4-6 Wochen & versenkt \\
\hline \multirow[t]{2}{*}{ Femur proximal } & Schrauben & 6-12 Monate & altersabhängig \\
\hline & ESIN & 6-12 Monate & altersabhängig \\
\hline \multirow[t]{2}{*}{ ECF } & K-Drähte & nach Fugenschluss & \\
\hline & Schrauben & nach Fugenschluss & \\
\hline Femurdiaphyse & ESIN & 4-6-(12) Monate & altersabhängig \\
\hline Fixateur extern & 6-12 Wochen & & \\
\hline \multirow[t]{3}{*}{ Femur distal } & K-Drähte & 4-6 Wochen & transkutan \\
\hline & K-Drähte & 6-10 Wochen & versenkt \\
\hline & ESIN & 4-6 Monate & antegrad \\
\hline Patella & Zuggurtung & 6 Monate & Adoleszente \\
\hline Tibia proximal & Schrauben & 3 Monate & \\
\hline \multirow[t]{2}{*}{ Tibiadiaphyse } & ESIN & 4-6 Monate & \\
\hline & Fixateur extern & 6-8 Wochen & \\
\hline \multirow[t]{4}{*}{ Tibia distal, OSG } & K-Drähte & 4-6 Wochen & versenkt \\
\hline & Fixateur extern & 6-8 Wochen & \\
\hline & Schrauben & 3 Monate & \\
\hline & Platte & 4-6 Monate & \\
\hline \multirow[t]{3}{*}{ Beckenring ventral } & Zuggurtung & 3-6 Monate & \\
\hline & Platte & 3-6 Monate & \\
\hline & Fixateur extern & 4-6 Wochen & \\
\hline Beckenring dorsal & Schrauben & 3-6 Monate & ISG-Fugen kreuzend \\
\hline Azetabulum & Schrauben & 3 Monate & Fugen kreuzend \\
\hline Wirbelsäule BWS/LWS & Fixateur interne & (3) -6 Monate & \\
\hline
\end{tabular}

gestellt und letztlich eine individuelle und gemeinsame Entscheidung für oder gegen die ME getroffen werden. Neben den bereits o.g. Risiken, wie den möglichen negativen Einfluss auf das Knochenwachstum und die Implantatkorrosion und deren Folgen, bestehen weitere Risiken und mögliche Komplikationen beim Belassen des Implantats:
- Ermüdungsbruch des Implantats bzw. am Implantatende

- Spätinfekt

- erschwerte Bedingungen bei erneutem Unfall mit Bruch des betroffenen Knochens und/oder des Implantats

- erschwerte Bedingungen bei einer später evtl. doch noch notwendigen Implantatentfernung durch Verwachsungen bzw. durch Überwachsen mit dem Knochen (speziell bei Titanimplantaten, ESIN)

- erschwerte Diagnostik und Therapie einer erneuten Fraktur

- Einschränkung später evtl. notwendiger diagnostischer Maßnahmen (CT, MRT)

Dem gegenüber stehen die Risiken bedingt durch die Implantatentfernung:

- intraoperative Frakturen

- Nervenläsionen

- Gefäßläsionen

- Nachblutung

- Hämatom/Serom

- Wundheilungsstörung

- Weichteilinfekt

- Knocheninfekt

- Gelenkinfekt

- Thrombembolie

- Verletzung der Wachstumsfuge mit ggf. hemmenden oder auch stimulierenden Effekten

Zusätzlich bestehen Spätkomplikationen die ebenfalls in die Entscheidung für eine mögliche Implantatentfernung einfließen müssen:

- Refraktur mit ggf. der Notwendigkeit einer erneuten Osteosynthese

- neue Fraktur an einer „Sollbruchstelle“ (z. B. Schraubenloch, ESI-Nageleintrittsstelle)

- Spätinfekt

- verstärkte Narbenbildung u.U. mit Narbenkorrektur

- Narbenkontraktur

- bleibende Funktionseinschränkung trotz ME z. B. im Gelenkbereich

\section{Planung}

Ist die gemeinsame Entscheidung zur Implantatentfernung beim Kind getroffen worden, erfolgt die Planung des Eingriffs und die entsprechende Operationsvorbereitung.

Folgende Überlegungen müssen im Vorfeld der ME angestellt und die daraus resultierenden Entscheidungen in die Planung miteinbezogen werden:

- Operationstermin/Zeitpunkt

- evtl.Zusatzmaßnahmen

- ambulanter oder stationärer Eingriff

- Art der Narkose

- postoperatives Management

- benötigtes Instrumentarium

Medizinisch gesehen ist die Implantatentfernung bei Vorliegen einer bewegungs- und belastungsstabilen Ausheilung der ursprünglichen knöchernen Verletzung möglich (Tab. 1). Der Termin hängt in der Praxis jedoch in aller Regel 


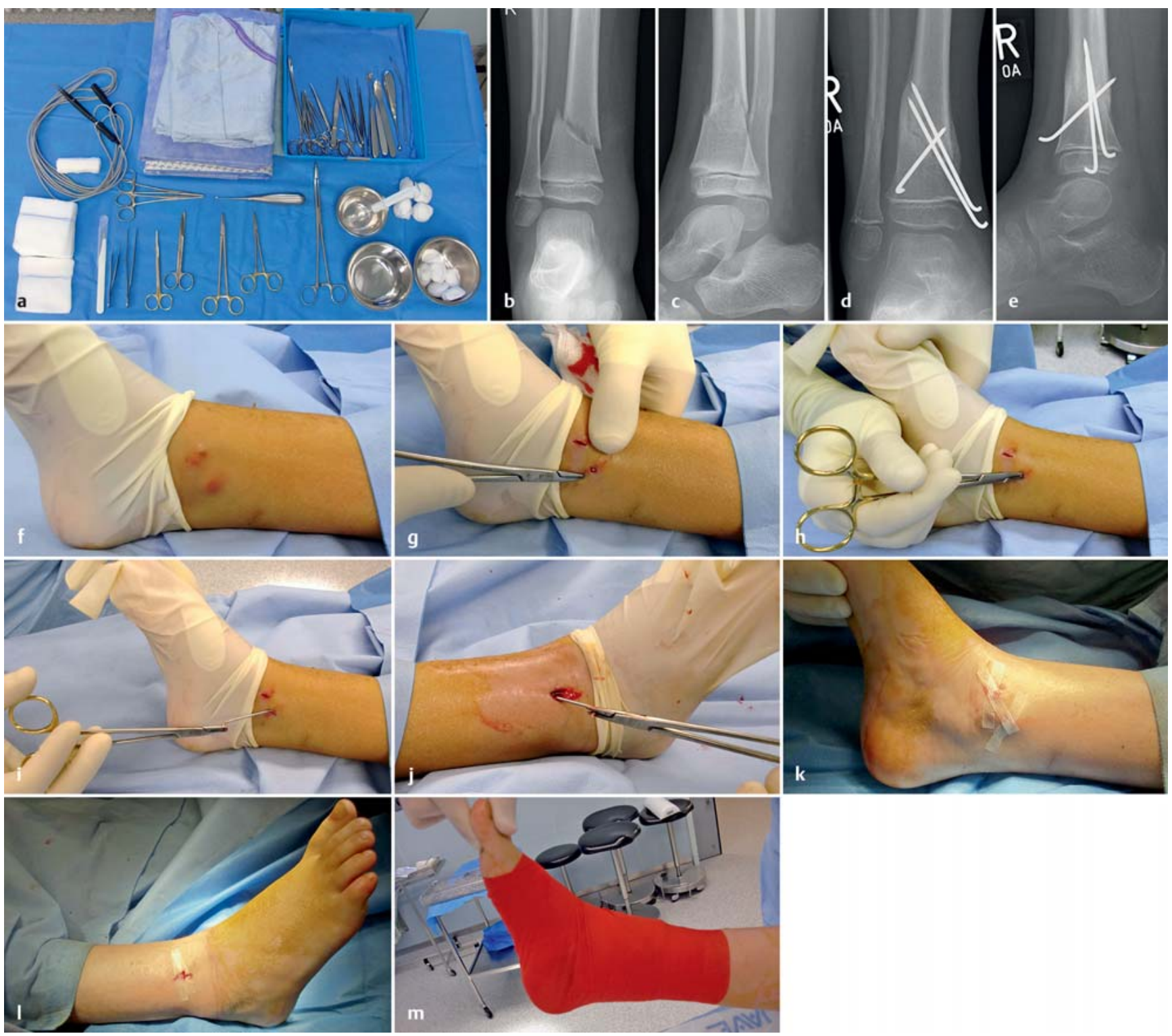

Abb. $\mathbf{1}$ a bis $\mathbf{m}$ Beispiel für die Zusammenstellung des OP-Instrumentariums für eine ME von K-Drähten mit OP-Ablauf am distalen Unterschenkel bei einem 7-jährigen Kind. a Instrumentiertisch. b-e präoperative Röntgenbilder (2 Ebenen). f-j Intraoperativer Ablauf. k-m Z. n. Hautverschluss und Wundverband.

vor allem auch von zeitlichen Möglichkeiten und Wünschen der Erziehungsberechtigten ab. Häufig werden Termine in den Schulferien, der prüfungsfreien Zeit oder innerhalb der Saisonpause der ausgeübten Sportart gewünscht. Die entsprechende OP-Kapazität ist ebenfalls ein möglicher begrenzender Faktor. In aller Regel ist bis auf noch einliegende K-Drähte, welche durch Bewegung des angrenzenden Gelenks auswandern können, oder ein noch nicht entfernter Fixateur externe, die Wiederaufnahme des Schulsports problemlos auch mit einliegendem Implantat möglich und der METermin über mehrere Wochen schiebbar. Nach Durchführung der üblichen präoperativen klinischen und radiologi- schen Untersuchungen einschließlich evtl. weiterer z.B. neurologischer Diagnostik (s.o.), kann die Indikation zur Durchführung zusätzlicher operativer Maßnahmen neben der Implantatentfernung bestehen. Darunter fallen Narbenkorrekturen, Neurolysen, Tenolysen, Arthrolysen aber auch Nervenersatzoperationen, Reosteosynthesen, Achskorrekturen sowie Operationen im Bereich der Wachstumsfugen in Form von Brückensprengungen bei partiellem vorzeitigem Fugenverschluss sowie auch temporäre Epi- oder Hemiepiphysiodesen zur Behebung von Achsabweichungen. Diese Zusatzmaßnahmen sollten möglichst im Rahmen der Implantatentfernung erfolgen und können den eigentlichen Ein- griff bez. der Dauer und des operativen Umfangs deutlich übertreffen. Dieser Umstand muss deshalb bei der internen Logistik berücksichtigt werden. Unter Umständen ist aufgrund der notwendigen Zusatzmaßnahmen die Durchführung des Eingriffs nicht im eigenen Haus möglich, und das Kind muss an ein Zentrum verwiesen werden.

\section{Durchführung}

Grundvoraussetzung für eine ambulante Implantatentfernung ist, dass die Erziehungsberechtigten im häuslichen Umfeld die Versorgung des Kindes gewährleisten können und die chirurgische Nachsorge durch den Operateur oder ei- 


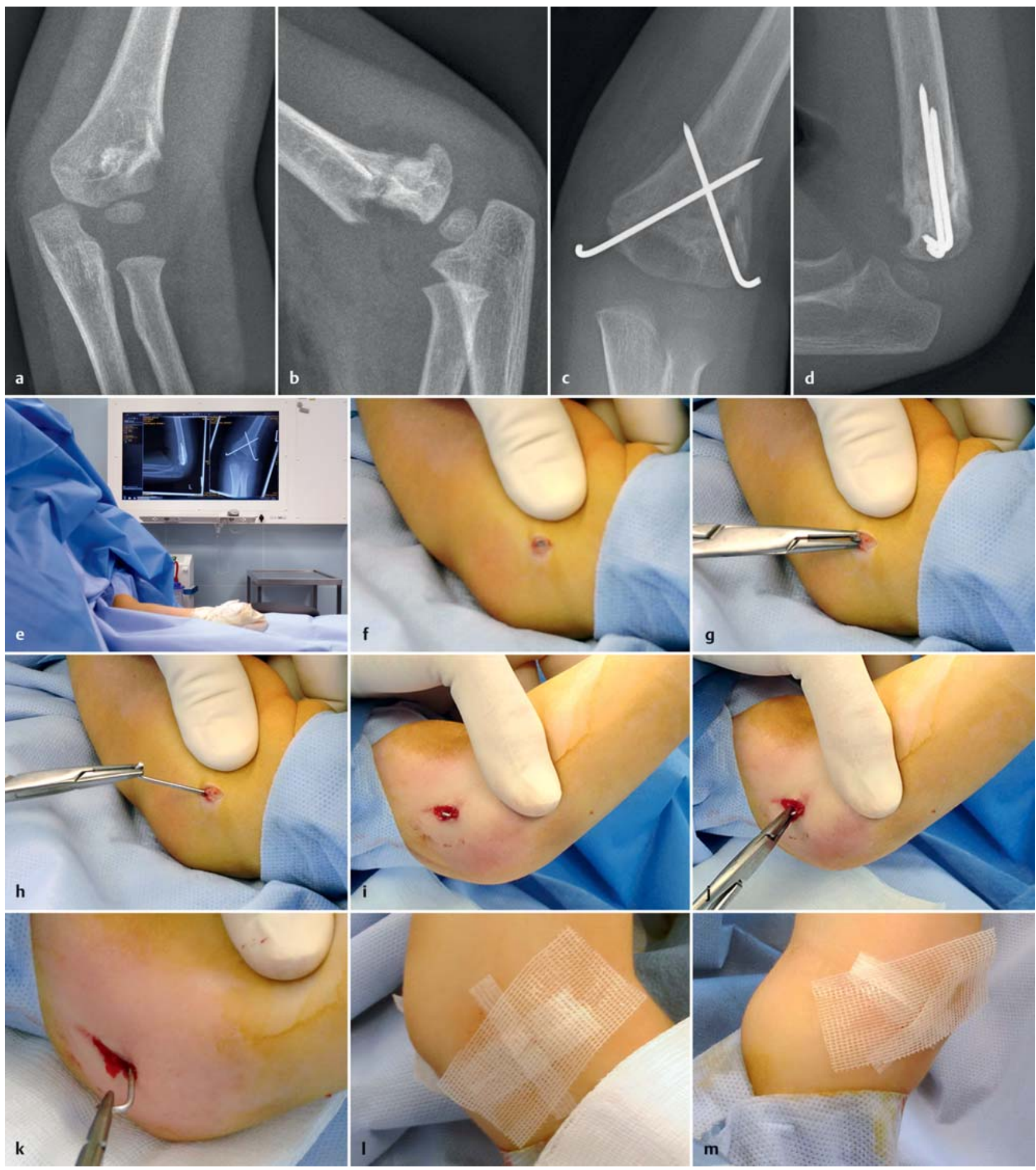

Abb. $\mathbf{2}$ a bis $\mathbf{m}$ Beispiel für eine K-Draht-Entfernung am distalen Humerus bei einem 3-jährigen Kind. a-d Präoperative Röntgenbilder (2 Ebenen). e Lagerung und Abdeckung. f-k Intraoperativer Ablauf. I, m Nicht klebende Wundauflage.

nen entsprechenden Vertreter in der Klinik oder auch im niedergelassenen Bereich orts- und zeitnah sichergestellt ist. Medizinisch gesehen können die meisten Implantatentfernungen ambulant durchgeführt werden. Dies gilt für $\mathrm{K}$ Draht- und isolierte Schraubenentfer- nungen, wie auch die Entfernung von ESIN und kleineren Plattenosteosynthesen. Die meisten stationären Implantatentfernungen bei Kindern sind zurückzuführen auf Eingriffe, bei denen größere Freilegungen notwendig sind, bei erhöhtem Operations- und Narkoserisiko und v.a. auch bei Wunsch der Eltern. Prinzipiell sollte selbst bei geplanter ambulanter Operation immer die Möglichkeit bestehen, bei entsprechendem Bedarf das Kind stationär überwachen $\mathrm{zu}$ können. Dies setzt bei der durchführenden Klinik die hierfür notwendige Kapa- 


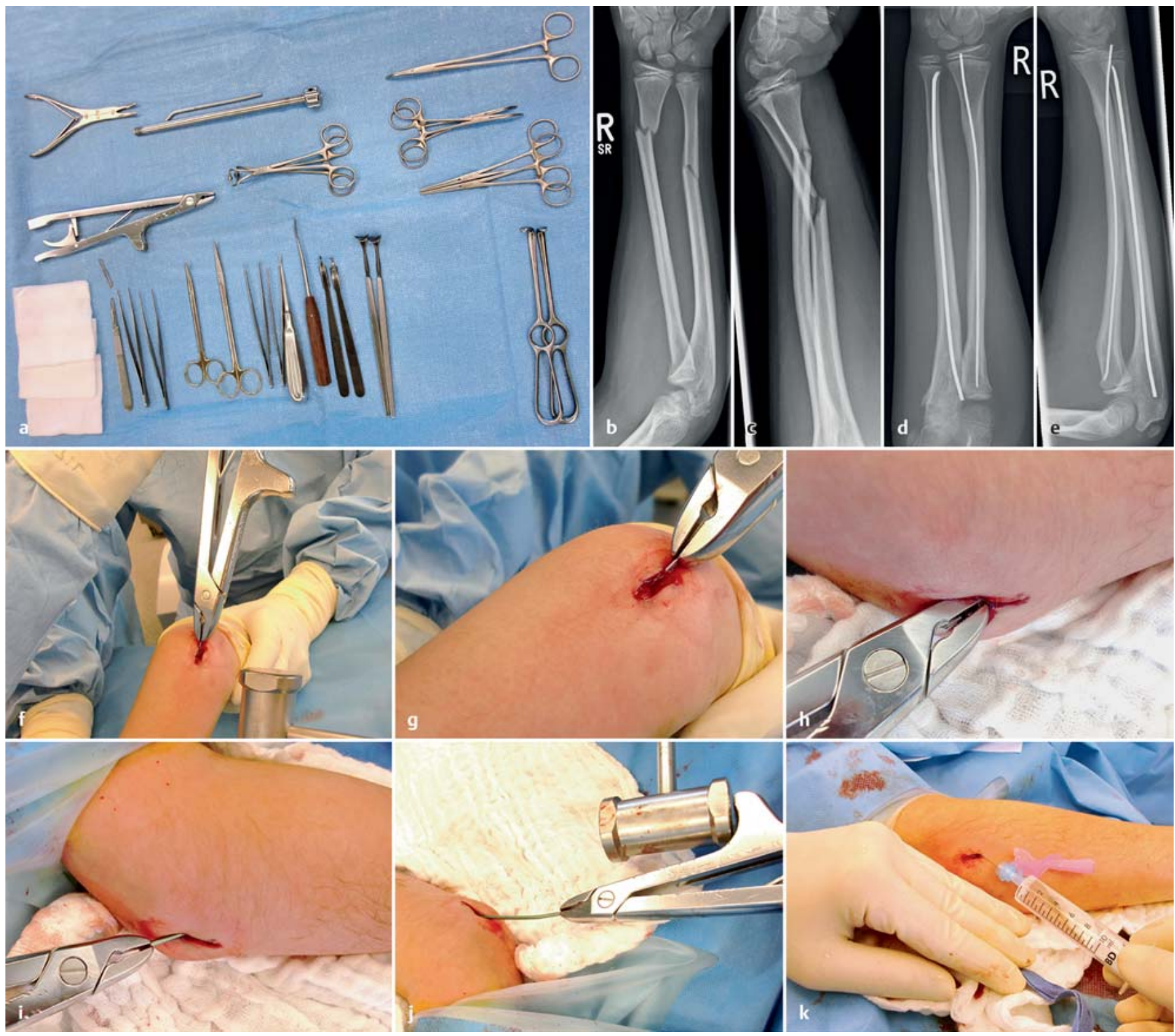

Abb. 3 a bis $\mathbf{k}$ Beispiel für die Zusammenstellung des OP-Instrumentariums für eine ME von ESI-Nägeln mit OP-Ablauf am Unterarm bei einem 10jährigen Kind. a Instrumentiertisch. b-e präoperative Röntgenbilder(2 Ebenen). f-j intraoperativer Ablauf.k Wundrandinfiltration mit Ropivacain.

zität und Logistik voraus (Kinderklinik, ggf. pädiatrische Intensivstation).

Bei Implantaten, die komplett unter der Haut versenkt sind, wird heute die Implantatentfernung im Rahmen einer kurzen Allgemeinnarkose mit Atemwegssicherung, üblicherweise über eine Larynxmaske, durchgeführt. Für die Eingriffe ist in aller Regel keine Relaxierung notwendig. Eine perioperative SingleShot-Antibiotikaprophylaxe ist bei elektiven Implantatentfernungen, bei denen keine größere Freilegung notwendig bzw. keine deutlich längere OP-Dauer als 30 Minuten zu erwarten ist, nicht zwingend erforderlich.
Es sind weiterhin im In- und Ausland transkutane K-Draht-Osteosynthesen mit freiliegenden K-Draht-Enden verbreitet. In diesen Fällen erfolgt die Implantatentfernung nur bei Bedarf mittels Analgosedierung ohne Anwesenheit der Anästhesie. Dies gilt meist auch bei den mit Fixateur externe ausbehandelten Frakturen. Bei K-Drähten wird teilweise auch eine ME in Lokalanästhesie beschrieben.

\section{OP-Instrumentarium}

Das Ziel ist es, das eingebrachte Implantat über den ursprünglichen Zugang, unter Vermeidung jeglicher Komplikation, zu entfernen. Hierfür bedarf es entsprechender Instrumente, die man am bes- ten als ME-Sieb für Kinder zusammenstellen sollte (Abb. 1, 2 und $\mathbf{3}$ ). Wenn mehrere geplante Eingriffen an einem Tag stattfinden, sollten diese Siebe in ausreichender Menge zur Verfügung stehen. In diesem Sieb sollten sämtliche für die gängigen Implantate (K-Draht, Kleinfragmentschraube, kanülierte Schraube, ESIN, Kleinfragmentplatte unterschiedlicher Dimension $(2,4,2,7,3,5 \mathrm{~mm})$ benötigten Instrumente, einschließlich Schraubendreher mit unterschiedlicher Größe für Sechskant- und Stardrive-Inbus, vorgehalten werden. Für sehr fest sitzende ESIN-Nägel ist es ratsam, einzeln steril eingeschweißte, fest arretierbare Fasszangen mit Ausschlagmöglichkeit auf Lager zu haben. Hin und wieder ist es erforderlich, knochenüberbaute 


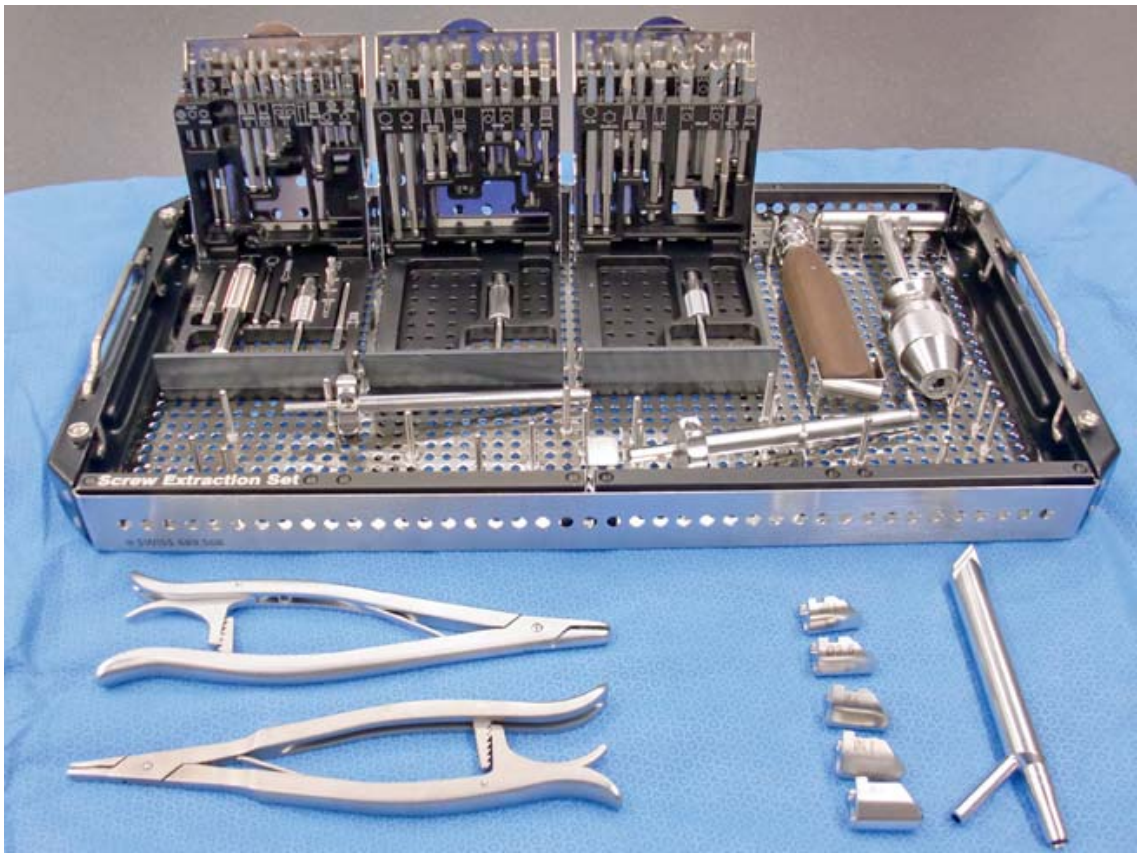

Abb. 4 Set für abgebrochene Schrauben (Schrauben-Extraktionsset, Fa. DePuy Synthes).

Implantate freizupräparieren, sodass Meißel auch in kleinen Größen im Sieb sein sollten. Unabdingbar ist das Vorhalten von entsprechenden Sets zur Entfernung abgebrochener Schrauben (Abb. 4). Bei den heute meist verwendeten Titanimplantaten, die auch bei Kindern z.T. winkelstabil implantiert werden, liegen regelmäßig wie auch bei den Erwachsenen, kaltverschweißte Schrauben oder Schraubenköpfe mit ausgeleiertem Inbus vor. Hierfür ist ein Sieb mit linksdrehenden Extraktionsschrauben, Hohlfräsen sowie linksdrehende Extraktionsbolzen jeweils in unterschiedlichen Dimensionen für Kleinfragment und Großfragment notwendig. Zusätzlich sind zum Aufbohren von kaltverschweißten Schraubenköpfen HSS- und Karbidbohrer (einzeln verschweißt, Einmalanwendung) mit entsprechender Möglichkeit zur Spülung und Absaugung der Bohrspäne, vorzuhalten.

\section{Vorbeugende Maßnahmen}

Schwierigen Implantatentfernungen kann man aber bereits bei der Osteosynthese vorbeugen. So ist darauf zu achten, dass die Implantate so platziert bzw. gekürzt und versenkt werden, dass bei deren Entfernung keine wesentlichen Schwierigkeiten auftreten sollten. Bei versenkten K-Draht-Enden macht es Sinn, diese nach dem Umbiegen relativ kurz abzuzwicken, ggf. vom Nerv weg (z.B.N. ulnaris bei der suprakondylären Humerusfraktur), und an die Knochenoberfläche anzubiegen. Hierdurch lassen sich bei der ME am ehesten Verletzungen der umliegenden Strukturen vermeiden. Das perkutane Herausstehenlassen von K-Drähten erleichtert die ME, welche dann meist mit deutlich geringerem Aufwand durchgeführt werden kann [5]. Aufgrund der jedoch damit zwingend notwendigen und aus unserer Sicht aufwendigen Ruhigstellung mit gipsfreiem Hof um die K-Drähte sowie die nicht auszuschließende Infektionsgefahr, führen wir dies an unserer Klinik nicht durch. Die Eintrittspunkte bei der ESINagelosteosynthese sollten immer im metaphysären Bereich liegen. Dies ermöglicht in aller Regel eine ausreichend lange extraossäre Strecke der ESIN, welche die einfache Anlage einer Fasszange bei der ME erleichtert. Die ESIN können in diesem Fall parallel zur Knochenoberfläche laufen und machen im Gegensatz zu den aufgebogenen ESIN-Enden meist keine Weichteilirritationen. Bei der ECF sollte man unbedingt Stahlimplantate verwenden, die deutlichen Schwierigkeiten bei der Entfernung von hier verwendeten Titanimplantaten sind hinlänglich bekannt [10]. Schrauben mit durchgedrehtem Inbus sollten sofort ausgetauscht werden, bei winkelstabilen Schrauben sind immer die dafür gedachten Drehmomentschraubendreher zu verwenden. Durch orthogrades Vorbohren durch die korrekt eingedrehten winkelstabilen Zielbohrbüchsen kann ein Kaltverschweißen der winkelstabilen Schrauben vermieden werden.

\section{Postoperatives Management/Nachsorge}

Eine generelle postoperative Röntgenkontrolle oder intraoperative Bildwandleruntersuchung zum Nachweis der vollständigen ME führen wir bei Entfernung isolierter K-Drähte, Schrauben oder ESIN bei unauffälligem OP-Verlauf nicht durch. Hierdurch wollen wir die Röntgenstrahlendosis bei den Kindern reduzieren. Auch dieses Vorgehen muss mit den Erziehungsberechtigten abgesprochen werden. Nach Entfernung größerer Plattenosteosynthesen oder bei technisch schwierigen Metallentfernungen führen wir eine postoperative Röntgenkontrolle am Tag der ME oder im Rahmen der ambulanten Nachkontrolle durch.

Nach der ambulanten wie auch stationären Implantatentfernung bei Kindern sollte ein strukturiertes postoperatives Management etabliert sein. Dies beinhaltet nach Möglichkeit einen kindgerechten, u.U. separaten Aufwachraum mit ausreichend Personal in der Klinik, in dem die Erziehungsberechtigten so früh wie möglich während der Aufwachphase am Bett des Kindes warten können (Abb. 5). Neben einer auf einem Stufenschema basierenden postoperativen Schmerztherapie sollte eine kindgerechte Tagesklinik bzw. Bettenstation mit entsprechend pädiatrisch/kinderchirurgisch ausgebildetem Personal zur Verfügung stehen. Bei ambulanten Implantatentfernungen kann das klinisch unauffällige Kind mit regelrechtem postoperativem Befund, gut eingestellter Analgesie nach Bedarf und nach erfolgter Nahrungsaufnahme von den Erziehungsberechtigten mitgenommen werden. Die Mitgabe eines Arztbriefs an den weiterbehandelnden Kollegen mit Vorgabe der Nachsorge (Wundkontrollen, Verbandswechsel, ggf. Fadenzug), der Mobilisation bzw. der Belastbarkeit der betroffenen Extremität und der Wiedersportfähigkeit einschließlich Schulsport, der wie Kontaktsport angesehen werden sollte, ist obligat. Es ist selbstverständlich, dass der Operateur dem Erziehungsberechtigten wie auch dem operierten Kind, persönlich von der Operation berichtet und die üblichen Fragen direkt beantwortet. So ist es in aller Regel möglich, 5 Tage nach durchgeführter ME bei kleinen Inzisionen die betroffene Stelle ohne Pflaster kurz abzuduschen, die Stelle sollte dann mit dem Handtuch nur trocken abgetupft werden. Baden und Schwimmen sind üblicherweise nach Abschluss der Wundheilung, also nach 


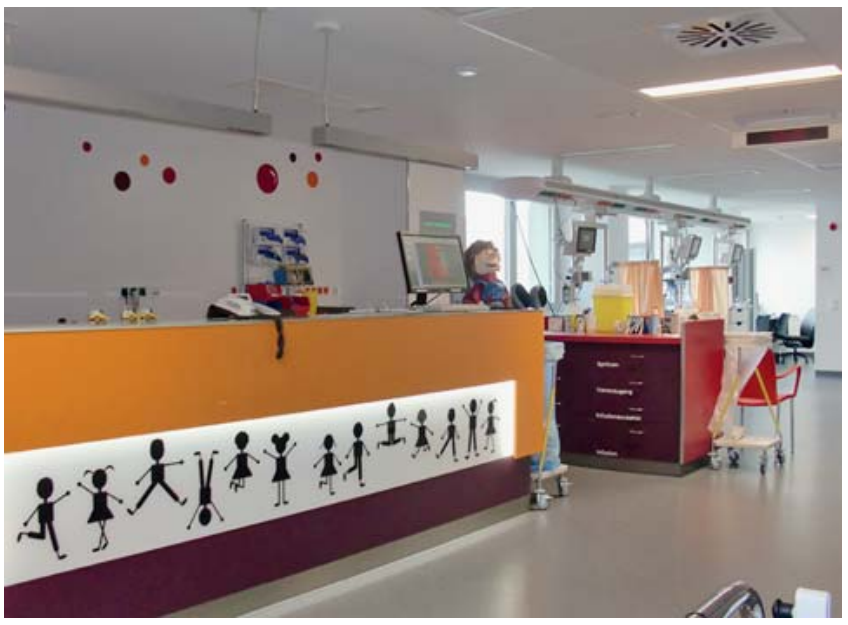

12-14 Tagen postoperativ möglich. Bei problemlosen Implantatentfernungen, bei denen präoperativ eine volle Funktion bzw. freie seitengleiche Beweglichkeit ohne Beschwerden und klinisch wie auch radiologisch ein unauffälliger Befund bestand, kann die Nachsorge des Kindes vom Kinderarzt, Hausarzt oder niedergelassenem Kollegen erfolgen. War dies nicht der Fall oder gab es u. U. Schwierigkeiten bei der Implantatentfernung, so ist die persönliche ambulante Nachbehandlung zu empfehlen. Aus unserer Erfahrung kann man durch eine ausführliche Aufklärung der Erziehungsberechtigten über die Operation den abschließenden Ablauf einschließlich der Nachsorge sowie durch einige, die Operation betreffende Maßnahmen die Gesamtsituation bei der Implantatentfernung bei Kindern deutlich vereinfachen. So ist vor dem Wundverschluss eine Wundrandinfiltration mit einem Lokalanästhetikum (z.B. Ropivacain, Wirkdauer 2-6 Stunden) sinnvoll. Hierdurch kann postoperativ die benötigte analgetische Therapie deutlich reduziert werden. Es ist ratsam, wenn immer möglich den Wundverschluss mit resorbierbarem Fadenmaterial durchzuführen. Somit entfällt der für Kinder extrem unangenehme Fadenzug, der manchmal deutlich mehr Aufwand als die eigentliche ME bereitet. Es sollten selbstklebende Pflaster vermieden werden. Wir verwenden standardisiert eine nicht verklebende sterile Wundauflage, darüber eine Kompresse, die dann angewickelt wird. Damit erspart man dem Kind eine weitere unangenehme Prozedur und sich oder dem weiterbehandelnden Kollegen viel „Geschrei“.

Die Frage, ab wann das Kind nach einer Implantatentfernung wieder in den Kin-
Abb. 5 Separater Kinderaufwachraum im Klinikum Augsburg.

dergarten oder die Schule gehen kann, ist in aller Regel eine individuelle Entscheidung der Eltern und des Kindes, weniger eine medizinische Vorgabe. Wenn das Kind den ambulanten Eingriff ohne Auffälligkeiten übersteht und die lokale Wund- und Schmerzsituation als problemlos eingestuft werden können, dann steht einem Schul- oder Kindergartenbesuch am 1. postoperativen Tag medizinisch nichts entgegen. Bei unserem Patientengut entscheiden die Eltern mit ihren Kindern meist, dass der 1 . Tag nach der Implantatentfernung zu Hause verbracht wird.

Demgegenüber sollte der Zeitpunkt, ab wann das Kind nach durchgeführter ME wieder Sport bzw. Schulsport betreiben kann, nach medizinischen Gesichtspunkten entschieden und entsprechend vorgegeben werden. Es sind auch hier immer individuelle Entscheidungen, die abhängig sind vom Patientenalter, der Lokalisation der ehemaligen Fraktur, der Art des entfernten Implantats sowie auch der betriebenen Sportart. So würde man bei einem Adoleszenten nach Entfernung einer Plattenosteosynthese mit mehreren Schrauben an der unteren Extremität die Wiedersportfähigkeit für Kontaktsport wie z. B. Fußball frühestens nach 4-6 Wochen wieder attestieren. Bei einem 5-jährigen Kind nach Entfernung zweier K-Drähte am distalen Radius ist meist jeglicher Sport wieder nach 2-3 Wochen möglich. Als bestehende Voraussetzungen der Wiedersportfähigkeit gelten für alle Kinder nach ME die abgeschlossene Wundheilung, die Beschwerdefreiheit sowie die volle Funktion der entsprechenden Extremität mit guter Beweglichkeit der angrenzenden Gelenke.

\section{Zusammenfassung}

Es gibt bei Kindern weder eine Evidenz für noch gegen die routinemäßige Implantatentfernung. Die Gründe für den Eingriff sind neben den auch bei Erwachsenen üblichen Umständen der implantatbedingten Einschränkung der Funktion, die lokale Reizung des umgebenden Gewebes und die Beeinflussung der Biomechanik, v.a. eine mögliche Beeinträchtigung des wachsenden Skeletts, die Implantatkorrosion und der Wunsch der Eltern. Dem gegenüber stehen sämtliche intra- und postoperativen operations- und narkosebedingte Risiken. Letztlich muss die Indikation zur Implantatentfernung bei Kindern immer individuell unter Abwägen des Nutzen-RisikoVerhältnisses gestellt und mit den Eltern besprochen werden. Um eine erfolgreiche Implantatentfernung bei Kindern durchführen zu können, bedarf es neben eines sorgsam ausgewählten ME-Instrumentariums einer standardisierten Planung, Durchführung und Nachsorge im Rahmen eines entsprechenden periund postoperativen Managements sowie eines möglichst kindgerechten Umfelds. Die beste Implantatentfernung bei Kindern ist und bleibt diejenige, die man nicht durchführen muss. Bei der Indikationsstellung zur operativen Versorgung einer Fraktur im Kindesalter sollte der behandelnde Arzt bei allen Frakturen, die auch konservativ gut zu behandeln sind, immer den Umstand, der in aller Regel noch anstehenden Implantatentfernung, bedenken. Die Erziehungsberechtigten müssen deshalb bereits zum Zeitpunkt der Entscheidung für oder gegen eine operative Frakturversorgung ausführlich darüber aufgeklärt werden, dass durch die Entscheidung zur Osteosynthese meist 2 Operationen mit $\mathrm{u}$. U. jeweils einer Narkose anstehen.

\section{Literatur}

${ }^{1}$ AWMF online. Leitlinien Unfallchirurgie, überarbeitete Leitlinie S1, 2014

2 Gorter EA, Vos DI, Sier CF et al. Implant removal associated complications in children with limb fractures due to trauma. Eur J Trauma Emerg Surg 2011; 37: 623-627

${ }^{3}$ Gras F, Marintschev I, Lenz M et al. Frakturen durch Materialentfernung. Trauma Berufskrankh 2014; 16 (Suppl. 4): S341-S348

${ }^{4}$ Hierholzer S, Hierholzer G. Osteosynthese und Metallallergie, Klinische Untersuchungen, Immunologie und Histologie des Implantatlagers. Traumatologie aktuell (Suppl. 1). Stuttgart: Thieme; 1991

5 von Laer L, Kraus R, Linhart WE. Frakturen und Luxationen im Wachstumsalter. 6. Aufl. Stuttgart, New York: Thieme; 2013

${ }^{6}$ Morshed S, Humphrey M, Corrales LA et al. Retention of flexible intramedullary nails fol- 
lowing treatment of pediatric femur fractures. Arch Orthop Trauma Surg 2007; 127 : 509-514

${ }^{7}$ Müller-Färber J. Die Metallentfernung nach Osteosynthesen. Orthopäde 2003; 32: 1039 1058

8 Ochs BG, Gonser CE, Baron HC et al. Refrakturen nach Entfernung von Osteosynthesematerialien. Unfallchirurg 2012; 115: 323-329

9 Perren SM. Evolution of the internal fixation of long bone fractures. The scientific basis of biological internal fixation: choosing a new balance between stability and biology. J Bone Joint Surg Br 2002; 84: 1093-1110

10 Raney EM, Freccero DM, Dolan LA et al. Evidence-based analysis of removal of orthopaedic implants in the pediatric population. J Pediatr Orthop 2008; 28: 701-704
11 Sinikumpu JJ, Keränen J, Haltia AM et al. A new mini-invasive technique in treating pediatric diaphyseal forearm fractures by bioresorbable elastic stable intramedullary nailing. Scand J Surg 2013; 102: 258-264

12 Takada N, Otsuka T, Suzuki H et al. Pediatric displaced fractures of the lateral condyle of the humerus treated using high strength, bioactive, bioresorbable F-u-HA/PLLA pins: a case report of 8 patients with at least 3 years follow-up. J Orthop Trauma 2013; 27: 281 284

${ }^{13}$ Unterer $N$. Verteilung von Abriebpartikeln von Titan und Stahl in verschiedene Organe. Eine vergleichende in-vivo Studie [Dissertation]. München: Ludwig-Maximilian-Universität; 2007

\section{Dr. med. Stefan Nuber}

Oberarzt

Klinik für Unfall-, Hand- und

Wiederherstellungschirurgie Klinikum Augsburg

Stenglinstraße 2

86156 Augsburg

stefan.nuber@klinikum-augsburg.de 